\title{
Preferência de Cotesia flavipes (Cameron, 1891) (Hymenoptera: Braconidae) por lagartas de Diatraea saccharalis (Fabricius, 1794) (Lepidoptera: Crambidae) alimentadas com diferentes cultivares de cana-de-açúcar ${ }^{1}$
}

\author{
Joseane Rodrigues de Souza², Arlindo Leal Boiça Júnior ${ }^{3}$, Dilermando Perecin ${ }^{4}$, \\ Jacqueline Tonielo da Costa ${ }^{5}$, Marília Lara Peixoto ${ }^{6}$ \\ http://dx.doi.org/10.1590/0034-737X201461060005
}

\begin{abstract}
RESUMO
O objetivo desta pesquisa foi avaliar a preferência do parasitoide Cotesia flavipes (Hymenoptera: Braconidae) por lagartas de Diatraea saccharalis (Lepidoptera: Crambidae), alimentadas com diferentes cultivares de cana-de-açúcar. O experimento foi conduzido em laboratório, em duas condições de alimentação, uma envolvendo lagartas de $D$. saccharalis, alimentadas em dieta artificial, e, a outra, com lagartas alimentadas em dieta artificial e mantidas temporariamente nos toletes dos cultivares de cana-de-açúcar. Os cultivares utilizados foram: SP80-1842 e SP81-3250, resistentes, e RB855536, suscetível à D. saccharalis. A preferência para oviposição das fêmeas de C. flavipes foi avaliada em testes com e sem chance de escolha, em delineamento inteiramente casualizado, com quatro tratamentos e 15 repetições. Lagartas de $D$. saccharalis com 19 dias de idade foram oferecidas para fêmeas de $C$. flavipes, acasaladas e com 24 horas de idade, em ambos os testes. No teste com chance de escolha, utilizou-se um olfatômetro, o qual constou de quatro compartimentos, em cujo centro liberaram-se quatro fêmeas de $C$. flavipes, enquanto, no teste sem chance de escolha, foram utilizadas placas de Petri, no interior das quais se colocou uma lagarta de D. saccharalis, oriunda das duas condições de alimentação. Nestes testes, foi observada a percentagem de parasitismo e, continuamente, o comportamento da primeira escolha de fêmeas de $C$. flavipes, em intervalos de zero a um, um a três, três a cinco, cinco a oito e de oito a dez minutos após as liberações. Lagartas de $D$. saccharalis, oriundas da alimentação em dieta artificial com colmos triturados dos cultivares, foram igualmente preferidas para atratividade de fêmeas do parasitoide $C$. flavipes. A percentagem de parasitismo de lagartas de $D$. saccharalis, criadas com dietas artificiais com colmos dos cultivares SP80-1042 e RB855536, foi igualmente parasitadas por C. flavipes.
\end{abstract}

Palavras-chave: Saccharum spp., resistência extrínseca, broca-da-cana, controle biológico, parasitoide.

\section{ABSTRACT}

\section{Preference of Cotesia flavipes (Cameron, 1891) (Hymenoptera: Braconidae) for larvae of Diatraea saccharalis (Fabricius, 1794) (Lepidoptera: Crambidae) fed on different sugarcane cultivars}

The objective of this study was to evaluate the preference of the parasitoid C. flavipes (Hymenoptera: Braconidae) for larvae of Diatraea saccharalis (Lepidoptera: Crambidae) fed on different sugarcane cultivars. The experiments

\footnotetext{
Recebido para publicação em 16/08/2012 e aprovado em 18/08/2014

Este trabalho é parte da tese de doutorado do primeiro autor, Instituição de Fomento - CAPES.

${ }^{2}$ Engenheira-Agrônoma, Doutora. Universidade Estadual do Maranhão, Centro de Estudos Supeirores de Balsas, Praça Joca Rego, s/n, Centro, 65800-000, Balsas, Maranhão, Brasil. joseaneagro@yahoo.com.br (Autora para correspondência).

3Engenheiro-Agrônomo, Doutor. Departamento de Fitossanidade, Universidade Estadual Paulista, Via de Acesso Professor Paulo Donato Castellane, s/n, 14884-900, Jaboticabal, São Paulo, Brasil. aboicajr@fcav.unesp.br

${ }^{4}$ Engenheiro-Agrônomo, Doutor. Departamento de Ciências Exatas, Universidade Estadual Paulista, Faculdade de Ciências Agrárias e Veterinárias, 14849-900, Jaboticabal, São Paulo, Brasil. perecin@fcav.unesp.br

${ }^{5}$ Engenheira-Agrônoma. Departamento de Solos e Adubos, Universidade Estadual Paulista, Via de Acesso Professor Paulo Donato Castellane, s/n, 14884-900, Jaboticabal, São Paulo, Brasil. jacquelinetonielo@yahoo.com.br

${ }^{6}$ Bióloga. Departamento de Fitossanidade, Universidade Estadual Paulista, Via de Acesso Professor Paulo Donato Castellane, s/n, 14884-900, Jaboticabal, São Paulo, Brasil. marilara19@yahoo.com.br
} 
were conducted in laboratory with two feeding conditions: larvae of $D$. saccharalis fed on artificial diet and larvae fed on artificial diet and kept temporarily on cane setts of the different cultivars. The resistant cultivars SP80-1842 and SP81-3250and the susceptible RB855536 cultivar to D. saccharalis were used in the experiment. The oviposition preference of $C$. flavipes females was evaluated in free-choice and no-choice tests, in a completely randomized design with four treatments and 15 replications. Larvae of D. saccharalis with 19 days of age were fed to mated females of $C$. flavipes with 24 hours of age in both tests.. For the free-choice test was used an olfactometer of four compartments, in which four females of $C$. flavipes were released in its center. For the no-choise test was used Petri dishes, each one with one larva of $D$. saccharalis treated under both feeding conditions. In these tests, it was determined the percentage of parasitism and continuously observed the behavior of the first choice of $C$. flavipes females at intervals of zero to one; one to three, three to five, five to eight and eight to ten minutes. Larvae of $D$. saccharalis reared in artificial diet with powdered stems of the cultivars were equaly preferred by females of the parasitoid $C$. flavipes; The percentage of parasitism of $D$. saccharalis larvae reared on artificial diets with powdered stems of the cultivars SP80-1042 and RB855536 were equaly parasitized by C. flavipes.

Key words: Saccharum spp., extrinsic resistance, sugarcane borer, biological control, parasitoid.

\section{INTRODUÇÃO}

Diatraea saccharalis (Fabricius, 1794) (Lepidoptera Crambidae) é um inseto- praga de grande importância econômica na cultura canavieira, em função dos sérios prejuízos oriundos do seu ataque, que contribuem para reduções significativas da produtividade agrícola e do aproveitamento industrial (DinardoMiranda, 2008).

A magnitude dos prejuízos causados pela $D$. saccharalis aos canaviais tem contribuído para o desenvolvimento de diferentes métodos para o controle dessa praga. Atualmente, o controle mais eficaz tem sido o biológico, por meio do endoparasitoide larval Cotesia flavipes (Cameron, 1891) (Hymenoptera: Braconidae) (Botelho \& Macedo, 2002; Pereira-Barros et al., 2005; Dinardo-Miranda, 2008).

O controle de $D$. saccharalis com o parasitoide $C$. flavipes dá-se por meio de liberações inundativas, que visam a interromper o crescimento populacional da broca e inicia-se com a localização do hospedeiro pelas fêmeas de $C$. flavipes. Para a localização do seu hospedeiro, fêmeas de $C$. flavipes utilizam estímulos olfativos para encontrar plantas infestadas por lagartas de $D$. saccharalis. Este apneumônio é uma substância hidrossolúvel, presente nas fezes secas ou reidratadas das lagartas de D. saccharalis. O contato com as fezes induz o inseto à procura, comportamento este caracterizado pela redução do ritmo de locomoção e por tateamento das fezes com as antenas (Botelho \& Macedo, 2002).
Além do controle biológico, outras medidas de controle de $D$. saccharalis podem ser adotadas, sendo em geral utilizadas de maneira integrada. Entre elas, destaca-se a utilização de cultivares resistentes, que tem sido apontada como um dos principais métodos de controle da broca-da-cana, por apresentar custos compatíveis com a cultura da cana-de-açúcar e por ser adequada à integração com o controle biológico (Botelho \& Macedo, 1998), sendo o seu emprego prática comum nos canaviais, pois auxilia na diminuição populacional da broca (Pinto et al., 2006).

Considerando-se as interações tritróficas (insetoplanta-inimigo natural), a classificação da resistência é dividida em duas categorias: intrínseca e extrínseca. A resistência intrínseca é aquela em que a planta defendese da praga diretamente, por meio de seus diversos mecanismos, enquanto a extrínseca ocorre quando a planta se beneficia dos inimigos naturais, influindo sobre eles e atuando, portanto, de forma indireta sobre a praga (Lara, 1991). A resistência de plantas a insetos e o controle biológico, em certos casos, podem ser antagonistas, sendo, dessa forma, importante que essa interação seja bem estudada, antes de esses métodos serem implementados a campo (Boiça Junior et al., 1997).

Em programas de controle biológico, é necessária a criação massal de inimigos naturais para sua posterior liberação em campo (Zanuncio et al., 2002). Para criação massal do parasitoide $C$. flavipes, é necessária, também, a criação do seu hospedeiro natural $D$. saccharalis, estando disponíveis, para tanto, inúmeras dietas artificiais (Parra \& Mihsfeldt, 1992). A qualidade dessas dietas 
artificiais é de fundamental importância para qualidade de hospedeiros produzidos (Vacari et al., 2012), o que poderá, também, influenciar na preferência do parasitoide.

Nesse sentido, quando se consideram as inter-relações inseto-planta, incluindo-se o terceiro nível trófico, poucas são as informações de estudos realizados acerca da resistência intrínseca de cultivares de cana-de-açúcar a D. saccharalis, e extrínseca, ao parasitoide $C$. flavipes (Boiça Junior et al., 1997; Silva, 2009).

Portanto, o objetivo deste trabalho foi avaliar a preferência de $C$. flavipes por lagartas de D. saccharalis, alimentadas em diferentes cultivares de cana-de-açúcar.

\section{MATERIAL E MÉTODOS}

O experimento foi conduzido no Laboratório de Resistência de Plantas a Insetos, localizado no Departamento de Fitossanidade, da Faculdade de Ciências Agrárias e Veterinárias - FCAV/UNESP, Campus de Jaboticabal, no período de novembro a dezembro de 2010 .

$\mathrm{Na}$ montagem do experimento, foram estabelecidas duas condições de alimentação, para a realização de testes de preferência para oviposição, com e sem chance de escolha, sendo elas: alimentadas em dieta artificial e alimentação em dieta artificial com, posteriormente, manutenção temporária em toletes de diferentes cultivares de cana-de-açúcar, durante um dia, antes da realização dos testes, para que o odor se tornasse próximo ao da condição natural de campo.

Os cultivares de cana-de-açúcar utilizados foram: SP80-1842 e SP81-3250, considerados resistentes (CTC, 2007), e RB855536, considerado suscetível a D. saccharalis (Coplana, 2009). A escolha dos cultivares deveu-se a sua vasta área plantada, no Estado de São Paulo, e foram adquiridos junto à Cooperativa dos Produtores de Cana do Oeste do Estado de São Paulo/ COPERCANA, localizada no município de Sertãozinho, $\mathrm{SP}$, enquanto as lagartas e os parasitoides foram fornecidos pelo Laboratório de Controle Biológico da Usina São Martinho, localizada no município de Pradópolis, SP.

Inicialmente, em área pertencente à Fazenda de Ensino e Pesquisa da FCAV/UNESP, toletes dos cultivares foram plantados em setembro de 2009, no sistema de sequeiro, em solo do tipo Latossolo Vermelho eutroférrico (LVef), de textura muito argilosa a moderada (Embrapa, 1999), visando à obtenção de colmos. Foi plantada uma linha de $15 \mathrm{~m}$ de cada cultivar, com espaço de $1 \mathrm{~m}$ no sulco de plantio. A adubação para a cultura da cana-de-açúcar foi realizada de acordo com as recomendações de Rossetto et al. (2008) e, os tratos culturais, segundo Xavier et al. (2008).
Quando as plantas atingiram 15 meses de idade, os colmos de cada cultivar foram coletados e levados para o laboratório, onde se retirou a parte central de cada colmo. Em seguida, eles foram cortados e colocados em estufa, a $60{ }^{\circ} \mathrm{C}$, por 48 h. Após a secagem, a parte central do colmo de cada cultivar foi triturada em moinho de facas modelo Willey TE-650, sendo, em seguida pesados $40 \mathrm{~g}$, que foram incorporados às dietas artificiais, conforme metodologia de Hensley \& Hammond (1968), modificada por Araújo et al. (1985). Foi preparada uma dieta de alimentação, que foi utilizada até que as lagartas de D. saccharalis atingissem 19 dias de idade, e, outra, de realimentação, cortada em forma de cubos e oferecida às lagartas parasitadas até a formação da massa cotonosa.

O delineamento experimental, utilizado em ambos os testes, foi inteiramente casualizado, com quatro tratamentos e 15 repetições. Os tratamentos foram constituídos a partir de dietas artificiais, com colmo seco triturado de cada cultivar de cana-de-açúcar, com exceção de uma delas, considerada dieta padrão.

Lagartas de D. saccharalis, com 19 dias de idade, com as fezes secas e reidratadas, foram oferecidas para fêmeas de $C$. flavipes, acasaladas e com 24 horas de idade, sendo, em seguida, mantidas em dieta de realimentação.

Para a realização do teste com chance de escolha, foi utilizado um olfatômetro, o qual constou de quatro compartimentos, com uma arena de $5 \mathrm{~cm}$ altura $\mathrm{x}$ 14,5 $\mathrm{cm}$ de diâmetro, revestida por papel filtro e coberta por uma tampa de acrílico. No centro do olfatômetro, foram liberadas quatro fêmeas de $C$. flavipes. Para a sucção do ar, foi conectado à base do olfatômetro um aparelho de inalação (Nevoni 6006), por meio de mangueiras de silicone (2,54 cm de diâmetro). As mangueiras de silicone foram fechadas com tecido do tipo 'voile', a fim de evitar a fuga das lagartas, conforme descrito por Angelini \& Boiça Junior (2009).

Primeiramente, foi realizado o teste com as lagartas sobre toletes correspondentes a cada cultivar e, no centro do olfatômetro, com o sistema desligado, foram liberadas as quatro fêmeas de $C$. flavipes, em cinco repetições. Os tratamentos (odores) foram posicionados ao acaso, em cada um dos compartimentos do olfatômetro. Ao ligar o olfatômetro, os odores provenientes dos quatro compartimentos misturaram-se e as fêmeas puderam-se movimentar livremente. Em seguida, realizou-se o teste em dieta, em que, em cada um dos compartimentos do olfatômetro, as lagartas foram colocadas sobre as dietas com que foram alimentadas, sendo o ensaio conduzido conforme a descrição citada anteriormente.

Após cada repetição, o olfatômetro era desmontado e limpado com álcool, sorteando-se, em seguida, a dis- 
posição dos tratamentos. Em ambos os ensaios, observou-se continuamente o comportamento da primeira escolha, em intervalos de um a três, três a cinco, cinco a oito e de oito a dez minutos, e o tempo que o parasitoide levou para atingir o compartimento do olfatômetro escolhido e para, assim, ovipositar nas lagartas de $D$. saccharalis. Além disso, foi avaliada também a percentagem de parasitismo.

No teste sem chance de escolha, utilizaram-se placas de Petri $(7 \mathrm{~cm} \times 1,5 \mathrm{~cm})$ com uma lagarta de $D$. saccharalis, com 19 dias de idade, oriunda da alimentação com dieta contendo colmo, correspondente a cada cultivar. Foi realizado também o teste sem chance de escolha, com lagartas alimentadas com dieta artificial e que foram mantidas temporariamente no tolete dos diferentes cultivares de cana-de-açúcar. A seguir, liberouse, no centro da placa, uma fêmea do parasitoide, sendo as avaliações realizadas conforme descrito no teste com chance de escolha, levando-se em consideração o tempo que o parasitoide levou para ovipositar nas lagartas de D. saccharalis.

Para os vários efeitos de interesse, foi realizada a análise de variância (teste F) e as médias foram comparadas pelo teste de Tukey, a 5\% significância , utilizando-se o programa SAS (SAS, 2004). Para comparar as frequências de escolha de fêmeas de $C$. flavipes, as médias foram comparadas, empregou-se a hipótese com e sem chance de escolha, nos intervalos de tempos descritos anteriormente, e interpretadas, para os vários efeitos de interesse, pelo teste exato de Fischer, e os respectivos "p-values", sendo considerados significativos os valores menores que 0,10 (10\%) (Beiguelman, 1996).

Os efeitos considerados foram: 1) efeito da chance, com que se comparou a média de escolha; 2) efeito da condição alimentar, com que se comparou a média da condição alimentar das lagartas, em dieta artificial e quando foram alimentadas nos toletes dos diferentes cultivares; 3 ) efeito de tratamento, com que se comparou a média entre os quatro tratamentos; 4) efeito da interação tratamentos x condição alimentar, com que se compararam os efeitos de tratamentos que são dependentes da condição alimentar; 5) efeito da interação chance x condição alimentar, com que se comparou o efeito da chance, que é dependente da condição alimentar; 6) efeito da condição alimentar - tratamentos x chance, com que se comparou a média entre os tratamentos, que é dependente da chance de escolha.

\section{RESULTADOS E DISCUSSÃO}

De acordo com as frequências observadas das fêmeas de $C$. flavipes para ovipositar em lagartas de $D$. saccharalis, com relação aos odores, tanto na escolha inicial quanto ao término do intervalo de minutos observados, os resultados revelaram, no teste com chance de escolha, efeito da interação entre os tratamentos, quando foi oferecida dieta artificial para as lagartas de D. saccharalis e quando foram oferecidos toletes dos diferentes cultivares. No entanto, observou-se ausência daquele efeito no teste sem chance de escolha, quando às fêmeas de $C$. flavipes foram oferecidas lagartas de D. saccharalis, oriundas da alimentação com dieta artificial (Tabela 1).

No teste com chance de escolha, quando lagartas de D. saccharalis, alimentadas e mantidas nos toletes dos cultivares foram oferecidas para fêmeas de C. flavipes, notaram-se diferenças significativas, com relação ao número de fêmeas do parasitoide que foram atraídas pelas lagartas de $D$. saccharalis, entre a testemunha versus cultivares, entre os tratamentos, e entre os cultivares RB855536 e SP81-3250. Esses resultados também foram observados quando lagartas de $D$. saccharalis, alimentadas com dieta artificial, foram oferecidas para fêmeas de $C$. flavipes (Tabela 1). Levando-se em consideração as 15 fêmeas de $C$. flavipes que foram liberadas, com relação à preferência de oviposição para a primeira escolha, e com base nos intervalos de minutos avaliados, a testemunha e o cultivar RB855536 não diferiram entre si e atraíram cinco fêmeas do parasitoide, enquanto os cultivares SP80-1842 e SP81-3250 atraíram apenas duas e uma fêmea, respectivamente, de acordo com as frequências observadas na Tabela 1. Considerando-se os mesmos intervalos de minutos e quando lagartas de D. saccharalis, alimentadas com dieta artificial, foram oferecidas para fêmeas de C. flavipes, observou-se, na testemunha, a atração de apenas uma fêmea do parasitoide, enquanto o cultivar SP81-3250 atraiu 12 fêmeas, o SP80-1842 atraiu nove e o RB855536 atraiu seis fêmeas de $C$. flavipes (Tabela 1).

No teste sem chance de escolha, constatou-se que, quando lagartas de $D$. saccharalis, alimentadas e mantidas temporariamente nos toletes, foram oferecidas para fêmeas de $C$. flavipes, observaram-se diferenças significativas entre a testemunha versus cultivares, entre os tratamentos e entre os cultivares SP80-1842 e RB855536. No entanto, quando lagartas de D. saccharalis, alimentadas com dieta artificial, foram oferecidas para fêmeas de $C$. flavipes, notaram-se apenas diferenças significativas entre os tratamentos (Tabela 1). O cultivar SP813250 atraiu sete fêmeas, enquanto RB855536 e SP801842 atraíram, cada um, uma fêmea do parasitoide. Quando lagartas de D. saccharalis, alimentadas com dieta artificial, foram oferecidas para fêmeas de C. flavipes, notou-se que o cultivar SP81-3250 atraiu nove fêmeas, enquanto os cultivares RB855536 e SP80-1842 atraíram oito fêmeas de $C$. flavipes (Tabela 1). 


\begin{tabular}{|c|c|c|c|c|c|c|c|c|c|c|c|c|c|}
\hline \multirow{3}{*}{ Tratamentos } & \multirow{3}{*}{$\begin{array}{l}\text { Condição } \\
\text { alimentar }\end{array}$} & \multicolumn{12}{|c|}{ Atratividade } \\
\hline & & \multicolumn{6}{|c|}{ Com Chance } & \multicolumn{6}{|c|}{ Sem Chance } \\
\hline & & 0 - 1' & $1-3$ & $3-5$ & $5-8$, & $8-10$ & Atraídas & 0 - 1' & 1 - 3' & $3-5$ & $5-8$ & 8 - 10' & Atraídas \\
\hline \multirow{2}{*}{ Testemunha (A) } & tolete & 0 & 2 & 1 & 1 & 1 & 5 & 0 & 0 & 0 & 0 & 0 & 0 \\
\hline & dieta & 0 & 0 & 1 & 0 & 0 & 1 & 0 & 0 & 0 & 0 & 0 & 0 \\
\hline \multirow{2}{*}{ SP80-1842 (B) } & tolete & 0 & 2 & 0 & 0 & 0 & 2 & 0 & 0 & 0 & 1 & 0 & 1 \\
\hline & dieta & 0 & 3 & 4 & 2 & 0 & 9 & 4 & 2 & 0 & 0 & 2 & 8 \\
\hline \multirow{2}{*}{ RB855536 (C) } & tolete & 0 & 2 & 2 & 1 & 0 & 5 & 1 & 0 & 0 & 0 & 0 & 1 \\
\hline & dieta & 0 & 1 & 2 & 1 & 2 & 6 & 3 & 3 & 1 & 0 & 0 & 8 \\
\hline \multirow{2}{*}{ SP81-3250 (D) } & tolete & 0 & 0 & 0 & 0 & 1 & 1 & 2 & 3 & 2 & 0 & 0 & 7 \\
\hline & dieta & 1 & 5 & 4 & 0 & 2 & 12 & 3 & 2 & 1 & 2 & 1 & 9 \\
\hline$\overline{A \text { vs } B, C \text { e } D^{1}}$ & tolete & \multicolumn{6}{|c|}{$0,0012 *$} & \multicolumn{6}{|c|}{$0.0056 *$} \\
\hline $\mathrm{A}$ vs $\mathrm{B}, \mathrm{C}$ e $\mathrm{D}^{1}$ & dieta & \multicolumn{6}{|c|}{$0,0315^{*}$} & \multicolumn{6}{|c|}{$0,4658^{\text {ns }}$} \\
\hline $\mathrm{A}, \mathrm{B}, \mathrm{C}$ e $\mathrm{D}^{1}$ & tolete & \multicolumn{6}{|c|}{$0,0012 *$} & \multicolumn{6}{|c|}{$0,0315^{*}$} \\
\hline $\mathrm{A}, \mathrm{B}, \mathrm{C}$ e $\mathrm{D}^{1}$ & dieta & \multicolumn{6}{|c|}{$0,4658^{\mathrm{ns}}$} & \multicolumn{6}{|c|}{$0,0560^{*}$} \\
\hline $\mathrm{B}, \mathrm{C}$ e $\mathrm{D}^{1}$ & tolete & \multicolumn{6}{|c|}{$0,1372^{*}$} & \multicolumn{6}{|c|}{$0,9526^{\mathrm{ns}}$} \\
\hline $\mathrm{B}, \mathrm{C}$ e $\mathrm{D}^{1}$ & dieta & \multicolumn{6}{|c|}{$0,1491^{*}$} & \multicolumn{6}{|c|}{$0,0282^{\text {ns }}$} \\
\hline $\mathrm{B}$ vs $\mathrm{C}^{1}$ & tolete & \multicolumn{6}{|c|}{$0,4079^{\mathrm{ns}}$} & \multicolumn{6}{|c|}{$1,0000^{*}$} \\
\hline $\mathrm{B}$ vs $\mathrm{C}^{1}$ & dieta & \multicolumn{6}{|c|}{$0,4287^{\mathrm{ns}}$} & \multicolumn{6}{|c|}{$1,0000^{\mathrm{ns}}$} \\
\hline $\mathrm{B}$ vs $\mathrm{D}^{1}$ & tolete & \multicolumn{6}{|c|}{$0,2141^{\mathrm{ns}}$} & \multicolumn{6}{|c|}{$0,8044^{\mathrm{ns}}$} \\
\hline $\mathrm{B}$ vs $\mathrm{D}^{1}$ & dieta & \multicolumn{6}{|c|}{$0,4828^{\mathrm{ns}}$} & \multicolumn{6}{|c|}{$0,0583^{\mathrm{ns}}$} \\
\hline $\mathrm{C}$ vs $\mathrm{D}^{1}$ & tolete & \multicolumn{6}{|c|}{$0,0615^{*}$} & \multicolumn{6}{|c|}{$0,8887^{\mathrm{ns}}$} \\
\hline $\mathrm{C}$ vs $\mathrm{D}^{1}$ & dieta & & & & & & & & & & & & \\
\hline
\end{tabular}

${ }^{1}$ p-value do teste exato de Fischer; *significativo pelo teste de Fischer a 10\% de significância; ${ }^{\text {ns }}$ não significativo, dentro dos intervalos de tempos estudados. 
O comportamento de $C$. flavipes, parasitando lagartas de D. saccharalis e Diatraea flavipennella (Box, 1931), foi observado por Silva (2009) em testes com chance de escolha. Segundo o autor, fêmeas de $C$. flavipes apresentaram capacidade de reconhecer os voláteis dos hospedeiros, demonstrando preferência, ou não, quando criadas no seu hospedeiro de origem.

A presença de odores, em plantas de tomate infestadas por Macrosiphum euphorbiae (Thomas, 1878) (Hemiptera: Aphididae) e Tetranychus evansi Baker \& Printchard (Acari: Tetranychidae), também influenciou a preferência do predador Cycloneda sanguinea (Linnaeus, 1793) (Coleoptera: Coccinellidae) para localizar as referidas presas (Sarmento et al., 2008).

No que se refere ao tempo gasto pelas fêmeas de $C$. flavipes, para a primeira escolha pelas lagartas de $D$. saccharalis, nas condições em que foram realizados os testes com e sem chance de escolha, os resultados mostraram ausência em todos os efeitos considerados (Tabela 2), evidenciando que os cultivares fornecem estímulos semelhantes da preferência de $C$. flavipes, o que significa que todos os cultivares apresentam resistência extrínseca a $D$. saccharalis e a $C$. flavipes, pois não agiram adversamente sobre o parasitoide, o que é benéfico quanto à integração dos dois métodos de controle. Para Lara (1991), geralmente é vantajosa a integração dos métodos de controle resistência ao inseto e contole biológico, pois há efeito positivo da planta sobre os inimigos naturais.

Quanto à percentagem de parasitismo de fêmeas de C. flavipes, os resultados mostraram diferenças significativas para o efeito da condição alimentar e para o contraste entre a condição alimentar e os tratamentos (Tabela 3). Nesse sentido, fêmeas de $C$. flavipes apresentaram maior percentagem de parasitismo das lagartas alimentadas com dieta elaborada a partir do cultivar SP801842. No entanto, esta não diferiu estatisticamente daquela do cultivar RB855536. A resistência do cultivar SP80-1842 a D. saccharalis não prejudicou o parasitoide, o que revela a adequação da resistência com o controle biológico.

Tabela 2. Tempo de atração (minutos) de Cotesia flavipes atraídas por lagartas de Diatraea saccharalis criadas em dieta artificial, contendo colmo seco triturado de cultivares de cana-de-açúcar, em testes com e sem chance de escolha. Temperatura: $28 \pm 1{ }^{\circ} \mathrm{C}$; Umidade relativa: $80 \pm 10 \%$; Fotofase: 14 horas. Jaboticabal, SP, 2010

\begin{tabular}{|c|c|c|}
\hline \multirow{2}{*}{ Tratamentos } & \multirow{2}{*}{ Condiçãoalimentar } & Tempo para atração (min.) \\
\hline & & Com chance \\
\hline \multirow{2}{*}{ Testemunha (A) } & tolete & $6,00 a^{1}$ \\
\hline & dieta & $3,80 \mathrm{a}$ \\
\hline \multirow{2}{*}{ SP80-1842 (B) } & tolete & $2,11 \mathrm{a}$ \\
\hline & dieta & $1,00 \mathrm{a}$ \\
\hline \multirow{2}{*}{ RB855536 (C) } & tolete & $3,50 \mathrm{a}$ \\
\hline & dieta & $2,40 \mathrm{a}$ \\
\hline \multirow{2}{*}{ SP81-3250 (D) } & tolete & $2,16 \mathrm{a}$ \\
\hline & dieta & $9,00 \mathrm{a}$ \\
\hline F (chance) & & $0,41^{\mathrm{ns}}$ \\
\hline F (condição alimentar) & & $2,06^{\mathrm{ns}}$ \\
\hline $\mathrm{F}$ (tratamento) & & $1,12^{\mathrm{ns}}$ \\
\hline F (tratamento $\mathrm{x}$ condição alimentar) & & $1,11^{\mathrm{ns}}$ \\
\hline $\mathrm{F}$ (chance $\mathrm{x}$ condição alimentar) & & $2,61^{\mathrm{ns}}$ \\
\hline $\mathrm{F}$ (tratamento $\mathrm{x}$ chance $)$ & & $0,47^{\mathrm{ns}}$ \\
\hline \multirow{2}{*}{ Tratamentos } & \multirow{2}{*}{ Condiçãoalimentar } & Tempo para atração \\
\hline & & Sem chance \\
\hline \multirow{2}{*}{ SP80-1842 (B) } & tolete & $3,25 \mathrm{a}$ \\
\hline & dieta & $4,00 \mathrm{a}$ \\
\hline \multirow{2}{*}{ RB855536 (C) } & tolete & $3,44 \mathrm{a}$ \\
\hline & dieta & $1,00 \mathrm{a}$ \\
\hline \multirow{2}{*}{ SP81-3250 (D) } & tolete & $2,28 \mathrm{a}$ \\
\hline & dieta & $3,55 \mathrm{a}$ \\
\hline$\overline{\mathrm{F} \text { (condição alimentar) }}$ & & $0,41^{\mathrm{ns}}$ \\
\hline $\mathrm{F}$ (tratamento) & & $0,20^{\mathrm{ns}}$ \\
\hline F (tratamento $\mathrm{x}$ condição alimentar) & & $0,28^{\mathrm{ns}}$ \\
\hline
\end{tabular}

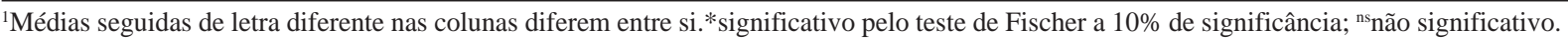


Tabela 3. Percentagem de parasitismo de Cotesia flavipes para oviposição em lagartas de Diatraea saccharalis criadas em dieta artificial, contendo colmo seco triturado de cultivares de cana-de-açúcar. Temperatura: $28 \pm 1{ }^{\circ} \mathrm{C}$; Umidade relativa: $80 \pm 10 \%$; Fotofase: 14 horas. Jaboticabal, SP, 2010

\begin{tabular}{lcc}
\hline Tratamentos & Condiçãoalimentar & Percentagem de parasitismo \\
\hline Testemunha (A) & tolete & $40,00 \mathrm{a}^{1}$ \\
& dieta & $80,00 \mathrm{a}$ \\
SP80-1842 (B) & tolete & $60,00 \mathrm{a}$ \\
& dieta & $86,67 \mathrm{a}$ \\
RB855536 (C) & tolete & $60,00 \mathrm{a}$ \\
& dieta & $66,67 \mathrm{ab}$ \\
SP81-3250 (D) & tolete & $60,00 \mathrm{a}$ \\
& dieta & $46,67 \mathrm{~b}$ \\
C.V. $(\%)$ & & 75,16 \\
F(condição alimentar) & & $6,12^{*}$ \\
F (tratamento) & & $1,89^{\text {ns }}$ \\
F (condição alimentar x tratamento) & $3,70^{*}$ \\
4) F (A vs B, C e D) & tolete & $3,63^{\text {ns }}$ \\
4) F (A vs B, C e D) & dieta & $2,07^{\text {ns }}$ \\
5) F (B, C e D) & tolete & $0,00^{\text {ns }}$ \\
5) F (B, C e D) & dieta & $6,21^{*}$ \\
\hline
\end{tabular}

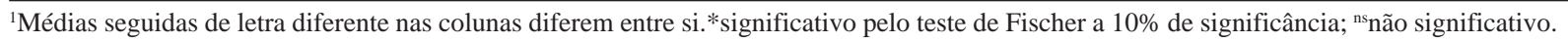

Boiça Junior et al., (1997) estudaram a influência de duas variedades de cana-de-açúcar (Saccharum spp.), incorporadas em dieta artificial, na forma de colmos triturados, no desenvolvimento de $D$. saccharalis e no parasitismo de $C$. flavipes e concluíram que a percentagem de parasitismo foi semelhante, para as lagartas criadas em dietas artificiais com colmos das variedades SP 71-1081 e SP 71-3146, consideradas moderadamente resistente e suscetível a $D$. saccharalis, respectivamente, demonstrando, também, a adequação da resistência com o controle biológico.

Lara et al., (1997) avaliaram a resistência de genótipos de sorgo, Sorghum bicolor, a Contarinia sorghicola (Coq.) e a D. saccharalis, e sua influência sobre os parasitoides Tetrastichus sp. e C. flavipes. Segundo os autores, os resultados para $C$. flavipes mostraram índices mais elevados de resistência nos genótipos BR-601 e 3 DX-57 e, menores, em BR-504, TX-2568, AF-28 e BR-501 e, nulos, em SC-3541 e TX-2567, concluindo que os dois primeiros genótipos mencionados apresentam resistência extrínseca à broca-da-cana e a C. flavipes na cultura do sorgo.

\section{CONCLUSÕES}

Lagartas de D. saccharalis, oriundas da alimentação com dieta artificial com colmos triturados dos cultivares, foram igualmente preferidas para atratividade das fêmeas do parasitoide $C$. flavipes.

A percentagem de parasitismo de lagartas de $D$. saccharalis, criadas com dietas artificiais com colmos dos cultivares SP80-1042 e RB855536, foram igualmente parasitadas por $C$. flavipes.

\section{AGRADECIMENTOS}

Os autores agradecem à COPERCANA, pelos cultivares utilizados na pesquisa; ao corpo técnico do Laboratório de Controle Biológico da Usina São Martinho, pelos insetos e pelo apoio em infraestrutura, e à Coordenação de Aperfeiçoamento de Pessoal de Nível Superior (CAPES), pela concessão da bolsa de estudos à primeira autora.

\section{REFERÊNCIAS}

Angelini MR \& Boiça Júnior AL (2009) Capacidade predatória e atratividade de Podisus nigrispinus (Dallas, 1851) (Hemiptera: Pentatomidae) por lagartas de Dione juno juno (Cramer, 1779) (Lepidoptera: Nymphalidae) criadas em folhas de genótipos de maracujazeiros. Revista Ceres, 56:25-30.

Araújo JR, Botelho PSM, Araújo SMSS, Almeida LC \& Degaspari N (1985) Nova dieta artificial para criação da Diatraea saccharalis (Fabr.) Saccharum, 36:45-48.

Beiguelman B (1996) Curso Prático de Bioestatística. 4ed. Ribeirão Preto, Sociedade Brasileira de Genética. 274p.

Boiça Junior AL, Lara FM \& Bellodi MP (1997) Influência de variedades de cana-de-açúcar, incorporadas em dieta artificial, no desenvolvimento de Diatraea saccharalis (Fabr.) e no seu parasitismo por Cotesia flavipes (Cam.) Anais da Sociedade Entomológica do Brasil, 26:537-542.

Botelho PSM \& Macedo N (2002) Cotesia flavipes para o controle de Diatraea saccharalis. In: Parra JRP, Botelho PSM, Corrêa-Ferreira BS \& Bento JMS (Eds.) Controle biológico no Brasil: parasitoides e predadores. São Paulo, Manole. p.409-421. 
Botelho PSM \& Macedo N (1998) Controle integrado da broca da canade-açúcar Diatraea saccharalis (Fabr. 1794) (Lepidoptera: Pyralidae) Brasil Açucareiro, 160:2-14.

Coplana (2009) Características agronômicas das variedades RB. Disponível em: < http//:www.coplana.com/gxpsites>. Acessado em: $20 \mathrm{de}$ março de 2009

CTC - Centro de tecnologia canavieira (2007) Recomendação de manejo das variedades. Disponível em: <http//:http:// www.ctcanavieira.com.br/var3g/pdfs/boletim_tecnico.pdf $>$. Acessado em: 20 de março de 2009.

Dinardo-Miranda LL (2008) Pragas. In: Dinardo-Miranda LL, Vasconcelos ACM \& Landell MGA (Ed.) Cana-de-açúcar. Campinas, Instituto Agronômico. p.350-362.

Embrapa - Empresa brasileira de pesquisa agropecuária (1999) Centro Nacional de Pesquisa de Solos. Sistema brasileiro de classificação de solos. Brasília, Embrapa solos. 412p.

Lara FM, Foss MRDA, Boiça Jr AL \& Trigo J G (1997) Resistência de genótipos de sorgo a Contarinia sorghicola (Coq.) (Diptera: Cecidomyiidae) e Diatraea saccharalis (Fabr.) (Lepidoptera: Pyralidae) e influência sobre parasitoides. Anais da Sociedade Entomológica Brasileira, 26:327-333.

Lara FM (1991) Princípios de resistência de plantas aos insetos. $2^{\mathrm{a}} \mathrm{ed}$. São Paulo, Ícone. 336p.

Parra JRP \& Mihsfeldt LH (1992) Comparison of artificial diets for rearing the sugarcane borer. In: Anderson, TE \& Leppla NC (Eds.) Advances in insect rearing for research and pest management. Boulder, Westview Press. p.195-209.

Pereira-Barros JL, Broglio-Micheletti SMF, Santos AJN, Carvalho LWT, Carvalho LHT \& Oliveira CJT (2005) Aspectos biológicos de Trichogramma galloi Zucchi, 1988 (Hymenoptera: Trichogrammatidae) criados em ovos de Diatraea saccharalis (Fabricius, 1794) (Lepidoptera: Crambidae). Ciência Agrotécnica, 29:714-718
Pinto AS, Garcia JF \& Oliveira HN (2006) Manejo das principais pragas da cana-de-açúcar. In: Segato SV, Pinto AS, Jendiroba E \& Nóbrega JCM (Eds.) Atualização em produção de cana-de-açúcar. Piracicaba, CP. p.257-280.

Rossetto R, Dias FLF \& Vitti AC (2008) Nutrição e adubação: fertilidade de solo, nutrição e adubação. In: Dinardo-Miranda LL, Vasconcelos ACM \& Landell MGA (Eds.) Cana-de-açúcar. Campinas, Instituto Agronômico. p.221-237.

Sarmento RA, Lemos F, Dias CR, Pallini A \& Venzon M (2008) Infoquímicos induzidos por herbivoria mediando a comunicação entre plantas de tomate e o predador Cycloneda sanguinea (Coleoptera: Coccinellidae). Revista Ceres, 55:439-444.

SAS Institute (2004) Statistical Analysis System user's guide. Version 9.1.3. Cary, North Carolina. 4420p.

Silva CCM (2009) Desempenho do parasitóide Cotesia flavipes (Cam.) (Hymenoptera: Braconidae) sobre Diatraea saccharalis (Fabr.) e Diatraea flavipennella (Box) (Lepidoptera: Crambidae). Dissertação de Mestrado. Universidade Federal Rural de Pernambuco, Recife. $51 \mathrm{p}$.

Vacari AM, Genovez GS, Laurentis VL \& De Bortoli AS (2012) Fonte proteica na criação de Diatraea saccharalis e seu reflexo na produção e no controle de qualidade de Cotesia flavipes. Bragantia, 71:355361.

Xavier MA, Mendonça JR \& Sanguino A (2008) Tecnologia de produção: Viveiros de mudas. In: Dinardo-Miranda LL, Vasconcelos ACM \& Landell MGA (Eds.) Cana-de-açúcar. Campinas, Instituto Agronômico. p.535-546.

Zanuncio JC, Guedes RNC, Oliveira HN \& Zanuncio TV (2002) Uma década de estudos com percevejos predadores: conquistas e desafios. In: Parra JRP, Botelho PSM, Corrêa-Ferreira BS, Bento JM (Eds.) Controle Biológico no Brasil - parasitóides e predadores. São Paulo, Manole. p.495-505. 